\title{
PKR inhibition mediates endotoxin tolerance in macrophages through inactivation of PI3K/AKT signaling
}

\author{
HAILIN XU*, JUAN CHEN*, XIANG SI, MINYING CHEN, FEI PEI, \\ CHUNFANG QIU, JIANFENG WU and XIANGDONG GUAN \\ Department of Critical Care Medicine, The First Affiliated Hospital of Sun Yat-sen University, \\ Guangzhou, Guangdong 510080, P.R. China
}

Received October 16, 2017; Accepted March 9, 2018

DOI: $10.3892 / \mathrm{mmr} .2018 .8869$

\begin{abstract}
Following long-term exposure to endotoxins, macrophages enter an immunosuppressive state that renders them unable respond to subsequent exposures to endotoxin, a phenomenon that is termed 'endotoxin tolerance'. Endotoxin tolerance increases the risks of secondary infection and mortality in patients with sepsis. In endotoxin-tolerant macrophages, the mixed variation of gene transcription is referred to as macrophage reprogramming. The mechanisms underlying macrophage reprogramming remain unclear at present. Interferon-induced double-stranded RNA-dependent protein kinase (PKR) is a widely expressed serine/threonine protein kinase. In addition to antiviral effects, PKR regulates the transcription of inflammatory cytokines by affecting transcription factors. However, the role of PKR in macrophage reprogramming remains to be elucidated. In the present study, the expression of inflammatory cytokines differed in lipopolysaccharide (LPS)-tolerant RAW264.7 macrophages compared with LPS-activated macrophages. Specifically, reverse transcription-quantitative polymerase chain reaction results demonstrated that the mRNA levels of tumor necrosis factor- $\alpha$, interleukin- $1 \beta$ (IL-1 $\beta$ ), C-X-C motif chemokine ligand 11, C-C motif chemokine ligand (CCL17), CCL22 and suppressor of cytokine signaling 3 were decreased, and mRNAs levels of arginase-1 (Arg1) and nitric oxide synthase 2 (iNOS) were increased, in LPS-tolerant macrophages compared with LPS-activated macrophages. Furthermore,
\end{abstract}

Correspondence to: Professor Jianfeng Wu and Professor Xiangdong Guan, Department of Critical Care Medicine, The First Affiliated Hospital of Sun Yat-sen University, 58 Zhongshan 2 Road, Guangzhou, Guangdong 510080, P.R. China

E-mail:wujianf@mail.sysu.edu.cn

E-mail: xiangdguan@126.com

*Contributed equally

Key words: macrophage, endotoxin tolerance, interferoninduced double-stranded RNA-dependent protein kinase, phosphatidylinositol-4,5-bisphosphate 3-kinase/AKT, sepsis, RAW264.7 western blot analysis demonstrated that the protein levels of phosphorylated (p)-PKR were significantly decreased in the LPS-tolerant cells. PKR activation with rotenone $(10 \mu \mathrm{M})$ abrogated endotoxin tolerance by increasing the levels of the IL-1 $\beta$, CCL17 and CCL22 mRNAs and decreasing the levels of the Arg1 and iNOS mRNAs. Furthermore, western blotting demonstrated that AKT was markedly inactivated in endotoxin-tolerant cells, as indicated by reduced p-AKT levels. However, levels of p-AKT were markedly increased following rotenone-induced PKR activation in endotoxin-tolerant cells. Ly294002 $(10 \mu \mathrm{M})$, a phosphatidylinositol-4,5-bisphosphate 3-kinase (PI3K)/AKT signaling inhibitor, partially reversed the rotenone-induced alleviation of endotoxin tolerance. These results demonstrated that PKR inhibition mediated endotoxin tolerance in macrophages, and these effects were partially mediated by PI3K/AKT signaling. PKR may be a potential target for the treatment of endotoxin tolerance in patients with sepsis.

\section{Introduction}

Innate immune cells, such as monocytes/macrophages, function in the defense against pathogens and the initiation and maintenance of the inflammatory response $(1,2)$. A robust inflammatory response is triggered when innate cells detect pathogens or their associated endotoxins, such as lipopolysaccharide (LPS), through pattern recognition receptors, including toll-like receptor 4 (TLR4), expressed on the cell surface $(3,4)$. However, macrophages are not able to respond to a subsequent challenge with LPS following long-term or repeated exposure to LPS. This phenomenon is termed 'endotoxin tolerance' $(5,6)$. The characterization of gene transcription following endotoxin tolerance revealed downregulation of certain genes upon LPS restimulation, including tumor necrosis factor- $\alpha$ (TNF- $\alpha$ ) (7), interleukin (IL)-1 $\beta$ (8), C-C motif chemokine ligand (CCL)17, CCL22 (9) and nitric oxide synthase 2 (iNOS) (10), while the expression of other genes, including chitinase-like 3 (Chil3) and arginase-1 (Arg1), was upregulated (11). The mixed transcriptional phenotype observed in tolerant cells indicates a gene reprogramming mechanism rather than a simple downregulation of LPS-induced gene expression $(5,12,13)$. The phenomenon of endotoxin tolerance has been observed in vitro and in vivo (14-16). In patients with sepsis, endotoxin 
tolerance has been reported to occur following inflammatory hypercytokinemia (17). Therefore, researchers previously hypothesized that endotoxin tolerance may be a mechanism used to protect the host against excessive inflammatory damage, as an uncontrolled inflammatory response leads to extensive tissue damage and septic shock (2). However, more recently, a different hypothesis has been formulated, which suggests that the endotoxin tolerant state is associated with secondary infection and may render the host more susceptible to septic progression and death (18). Therefore, strategies for the prevention of endotoxin tolerance may represent an effective treatment for sepsis (19).

Although endotoxin tolerance has been observed for $>50$ years (20), the mechanisms underlying macrophage reprogramming remain unclear. Overexpression of certain regulators in the TLR4 pathway, including IL-1 receptor-associated kinase-M (IRAK-M), SH2-containing inositol-5'-phosphatase and IRAK-M inducer hypoxia-inducible factor- $1 \alpha$, was previously reported to be implicated in the pathological process of endotoxin tolerance $(14,21,22)$. Among these regulators, interferon-induced double-stranded RNA-dependent protein kinase (PKR) was investigated in the present study. PKR is a widely expressed serine/threonine protein kinase (23). It is activated by multiple stimuli, including the inflammatory cytokines interferon and TNF- $\alpha$ (24), bacterial infection and viral double-stranded RNA (25-27). In addition to its antiviral properties, phosphorylated (p)-PKR also affects multiple transcription factors by activating numerous signaling pathways. These transcription factors, including interferon regulatory factor 3 (28) and nuclear factor $-\kappa \mathrm{B}(\mathrm{NF}-\kappa \mathrm{B})(29,30)$, are required for the expression of genes encoding inflammatory cytokines (25). However, the role of PKR in macrophage reprogramming remains to be elucidated. In the present study, the role of PKR in endotoxin tolerance was determined. In addition, the associated signaling pathways through which PKR may mediate macrophage reprogramming were also investigated.

\section{Materials and methods}

Cells and reagents. LPS (cat. no. L2654) and LY294002 (cat. no. L9908) were purchased from Sigma-Aldrich (Merck KGaA, Darmstadt, Germany). Rotenone (cat. no. 557368) was purchased from Millipore (Merck KGaA). RAW264.7 cells were purchased from the Type Culture Collection of the Chinese Academy of Sciences (Shanghai, China) and maintained in Dulbecco's modified Eagle's medium (DMEM; Thermo Fisher Scientific, Inc., Waltham, MA, USA) containing 10\% fetal bovine serum (Thermo Fisher Scientific, Inc.). Cells were maintained in a $5 \% \mathrm{CO}_{2}$ humidified incubator at $37^{\circ} \mathrm{C}$. Cell Counting Kit-8 (CCK-8) was obtained from Dojindo Molecular Technologies, Inc. (Kumamoto, Japan). Primary antibodies against AKT (cat. no. 4691S; rabbit), p-AKT (Thr308; cat. no. 13038S; rabbit) and $\beta$-actin (cat. no. 4970S; rabbit), and the anti-rabbit IgG, HRP-linked Antibody (cat. no. 7074S), were purchased from Cell Signaling Technology, Inc. (Danvers, MA, USA). The PKR antibody (cat. no. sc-708; rabbit) was purchased from Santa Cruz Biotechnology, Inc. (Dallas, TX, USA). The p-PKR antibody (T446; cat. no. ab32036; rabbit) was purchased from Abcam (Cambridge, UK). The primers for IL-1 $\beta$, CCL17, CCL22, Arg1, iNOS, TNF- $\alpha$, suppressor of cytokine signaling 3 (Socs3), C-X-C motif chemokine ligand 11 (CXCL11) and $\beta$-actin were supplied by Sangon Biotech Co., Ltd. (Shanghai, China). The Eastep Super Total RNA Extraction kit, GoScript Reverse Transcription System and GoTaq qPCR Master Mix were purchased from Promega Corporation (Madison, WI, USA).

Cell viability assays. Cell viability was measured using the CCK-8 assay according to the manufacturer's protocol. Briefly, RAW264.7 cells were seeded in 96-well culture plates at a density of 5,000 cells/well in DMEM and incubated in a humidified incubator at $37^{\circ} \mathrm{C}$ overnight. Cells were exposed to different concentrations of $\operatorname{LPS}(0,1,10,100,500$ and $1,000 \mathrm{ng} / \mathrm{ml}$ ) for $24 \mathrm{~h}$. After a $24 \mathrm{~h}$ incubation with LPS, $10 \mu \mathrm{l}$ CCK-8 reagent was added to each well and incubated for $1 \mathrm{~h}$. Subsequently, the optical density (OD) was measured at a wavelength of $450 \mathrm{~nm}$. The percentage of viable cells was determined using the following formula: Ratio $(\%)=[\mathrm{OD}$ (treated)-OD (blank)/OD (control)-OD (blank)] x100. Cell viability data are presented as the mean \pm standard error of the mean of three independent experiments, each containing three replicates.

Endotoxin tolerant model in RAW264.7 cells. The endotoxin tolerance model was established as follows. RAW264.7 cells were seeded in 6-well culture plates at a density of $5 \times 10^{5}$ cells/well in DMEM and incubated in a humidified incubator at $37^{\circ} \mathrm{C}$ overnight. Subsequently, cells were initially stimulated with medium alone or medium containing LPS (100 ng/ml) for $20 \mathrm{~h}$, washed with PBS twice and restimulated with medium or LPS (100 ng/ml) for $4 \mathrm{~h}$ prior to Reverse transcription-quantitative polymerase chain reaction (RT-qPCR) or $2 \mathrm{~h}$ prior to western blot analysis. Different durations of the second LPS stimulation were because expression of inflammatory cytokines depended on the activation of regulators and signaling $(9,31)$. Rotenone $(10 \mu \mathrm{M})$ was added $1 \mathrm{~h}$ before the second LPS stimulation and remained until the cells were lysed. LY294002 was used $2 \mathrm{~h}$ before the second LPS stimulation at a concentration of $10 \mu \mathrm{M}$ when necessary and lasted until the end of the second LPS stimulation. Macrophages that were continually cultured in DMEM were designated medium/medium (M/M), cells that were stimulated with LPS following the incubation with DMEM were designated medium/LPS (M/L) and cells that were restimulated with LPS following stimulation with the same dose of LPS were designated LPS/LPS (L/L). The cells were incubated in a humidified incubator at $37^{\circ} \mathrm{C}$ during the whole experimental process.

ELISA. TNF- $\alpha$ levels in the supernatants were analyzed using the TNF- $\alpha$ ELISA kit (F11630; Westang BioTechnology Corporation Ltd., Shanghai, China), according to the manufacturer's protocol. In brief, medium in the 6-well plate was pipetted into the 96 -wells plate directly. During the first incubation, TNF- $\alpha$ bound the capture antibody. Following washing, a detection antibody was added to the wells, which bound to the TNF- $\alpha$ immobilized during the first incubation. Subsequently, a horseradish peroxidase (HRP) conjugate was added to bind to the detection antibody. Finally, a substrate 
Table I. Primer sequences used for reverse transcription-quantitative polymerase chain reaction.

Primer sequence $\left(5^{\prime} \rightarrow 3^{\prime}\right)$

\begin{tabular}{lll}
\cline { 2 - 3 } Gene & \multicolumn{1}{c}{ Forward } & \multicolumn{1}{c}{ Reverse } \\
\hline TNF- $\alpha$ & GACGTGGAACTGGCAGAAGAG & TTGGTGGTTTGTGAGTGTGAG \\
IL-1 $\beta$ & GCAACTGTTCCTGAACTCAACT & ATCTTTTGGGGTCCGTCAACT \\
CXCL11 & GGCTTCCTTATGTTCAAACAGGG & GCCGTTACTCGGGTAAATTACA \\
CCL17 & GACGACAGAAGGGTACGGC & GCATCTGAAGTGACCTCATGGTA \\
CCL22 & ATTCTGTGACCATCCCCTCAT & TGTATGTGCCTCTGAACCCAC \\
Socs3 & TGCAGGAGAGCGGATTCTAC & AGCTGTCGCGGATAAGAAAG \\
Arg1 & CTCCAAGCCAAAGTCCTTAGAG & AGGAGCTGTCATTAGGGACATC \\
iNOS & GACGAGACGGATAGGCAGAG & CTTCAAGCACCTCCAGGAAC \\
-actin & GTGCTATGTTGCTCTAGACTTCG & ATGCCACAGGATTCCATACC \\
\hline
\end{tabular}

TNF- $\alpha$, tumor necrosis factor- $\alpha$; IL-1 $\beta$, interleukin-1 $\beta$; CXCL11, C-X-C motif chemokine ligand 11; CCL, C-C motif chemokine ligand; Socs3, suppressor of cytokine signaling 3; Arg1, arginase-1; iNOS, nitric oxide synthase 2.

solution was added and converted by the enzyme to a detectable form. The intensity of the colored product reflected the concentration of TNF- $\alpha$.

Preparation of whole-cell protein lysates. Cells were washed twice with ice-cold PBS and suspended in RIPA lysis buffer (P0013B; Beyotime Institute of Biotechnology, Haimen, China) containing $1 \mathrm{mM}$ phenylmethanesulfonyl fluoride and $1 \mathrm{mM}$ phosphatase inhibitors, and were centrifuged at $16,000 \mathrm{x} \mathrm{g}$ for $10 \mathrm{~min}$ to remove nuclei and cell debris. Supernatants were rapidly frozen at $-80^{\circ} \mathrm{C}$ or immediately used in western blot assays.

Western blot analysis. Protein concentrations were determined using the Pierce BCA Protein Assay kit (Thermo Fisher Scientific, Inc.) and $15 \mu \mathrm{g}$ cellular proteins were electroblotted onto polyvinylidene difluoride membranes following separation with $10 \%$ SDS-PAGE. The membranes were blocked for 15 min with QuickBlock Blocking Buffer for Western Blot (Beyotime Institute of Biotechnology, Haimen, China) at room temperature, followed by an overnight incubation at $4^{\circ} \mathrm{C}$ with primary antibodies against PKR, p-PKR, AKT, p-AKT and $\beta$-actin at a 1:1,000 dilution. Blots were washed three times with TBS/0.2\% Tween-20 (TBST) prior to incubation with the HRP-conjugated secondary antibody $(1: 5,000)$ for $1 \mathrm{~h}$ at room temperature. Blots were washed three times with TBST prior to development by enhanced chemiluminescence using the Immobilon Western Chemiluminescent HRP Substrate (Merck $\mathrm{KGaA}$ ). Band intensities were quantified using Quantity One software version 4.6.2 (Bio-Rad Laboratories, Inc., Hercules, CA, USA). $\beta$-actin was used as a loading control for whole-cell protein lysates.

RT-qPCR assays. Total RNA was extracted using the Eastep Super Total RNA Extraction kit, according to the manufacturer's protocol. A total of $1 \mu \mathrm{g}$ RNA was reverse transcribed into cDNAs using the GoScript Reverse Transcription System, including elongation at $42^{\circ} \mathrm{C}$ for $15 \mathrm{~min}$ and inactivation of reverse transcriptase at $70^{\circ} \mathrm{C}$ for $15 \mathrm{~min}$. qPCR was performed using GoTaq qPCR Master Mix. In brief, denaturation was performed at $95^{\circ} \mathrm{C}$ for $10 \mathrm{~min}$, annealing at $60^{\circ} \mathrm{C}$ for $1 \mathrm{~min}$, and elongation at $95^{\circ} \mathrm{C}$ for $15 \mathrm{sec}$ for 40 cycles. PCR was carried out in triplicate and using the Bio-Rad CFX96 instrument (Bio-Rad Laboratories, Inc.). Data were processed using Bio-Rad CFX manager version 3.1 (Bio-Rad Laboratories, Inc.). The housekeeping gene $\beta$-actin was used as the internal control. The relative expression levels were calculated using the $2^{-\triangle \Delta C q}$ method (32). The primer pairs used for qPCR are presented in Table I.

Statistical analysis. Prism 6 software (GraphPad, La Jolla, CA, USA) was used for statistical analysis. All data are presented as the mean \pm standard error of the mean $(n=3$ independent experiments). Data were analyzed using an unpaired two-tailed Student's t-test or one-way analysis of variance followed by a Tukey's multiple comparison test. $\mathrm{P}<0.05$ was considered to indicate a statistically significant difference.

\section{Results}

LPS promotes cell proliferation in a dose-depended manner. The viability of RAW264.7 cells was determined using the CCK-8 assay. As demonstrated in Fig. 1A, treatments with different concentrations of LPS $(1,10,100,500$ and $1,000 \mathrm{ng} / \mathrm{ml}$ ) significantly promoted cell proliferation compared with the control group. At LPS concentrations $<500 \mathrm{ng} / \mathrm{ml}$, cells proliferated in a concentration-dependent manner (Fig. 1A). No obvious cytotoxicity was observed when cells were treated with LPS at concentrations of $1-1,000 \mathrm{ng} / \mathrm{ml}$ (Fig. 1A).

$T N F-\alpha$ levels are decreased in $L / L$ macrophages compared with $M / L$ macrophages. Cells were cultured and stimulated with LPS using the methods described above. Supernatants were collected and examined using ELISA. TNF- $\alpha$ levels were demonstrated to be significantly reduced in LPS-tolerant L/L macrophages compared with LPS-activated M/L macrophages (Fig. 1B). 

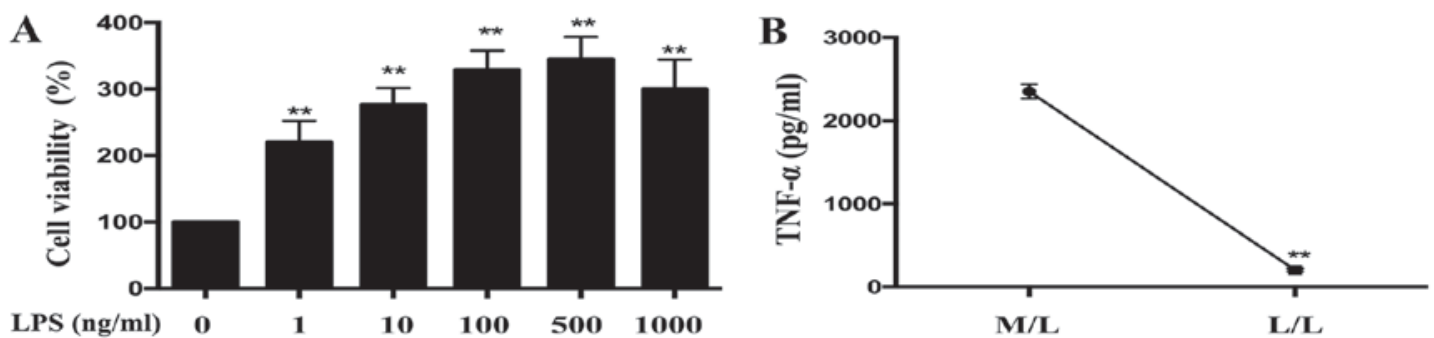

Figure 1. Effects of LPS stimulation on the cell viability and TNF- $\alpha$ expression of RAW264.7 macrophages. (A) Increasing concentrations of LPS promoted the proliferation of macrophages, as demonstrated using the Cell Counting Kit- 8 assay. ${ }^{* *} \mathrm{P}<0.01 \mathrm{vs} .0 \mathrm{ng} / \mathrm{ml}$ LPS group. Data represent the results from three independent experiments. (B) ELISA results demonstrated a decrease in the TNF- $\alpha$ level in LPS-tolerant L/L macrophages compared with LPS-activated $\mathrm{M} / \mathrm{L}$ macrophages. ${ }^{* *} \mathrm{P}<0.01 \mathrm{vs.} \mathrm{M} / \mathrm{L}$ cells. Data represent the results from three independent experiments. LPS, lipopolysaccharide; TNF- $\alpha$, tumor necrosis factor- $\alpha ; M / L$, initial incubation with medium followed by LPS stimulation; L/L, initial incubation with LPS followed by restimulation with LPS.
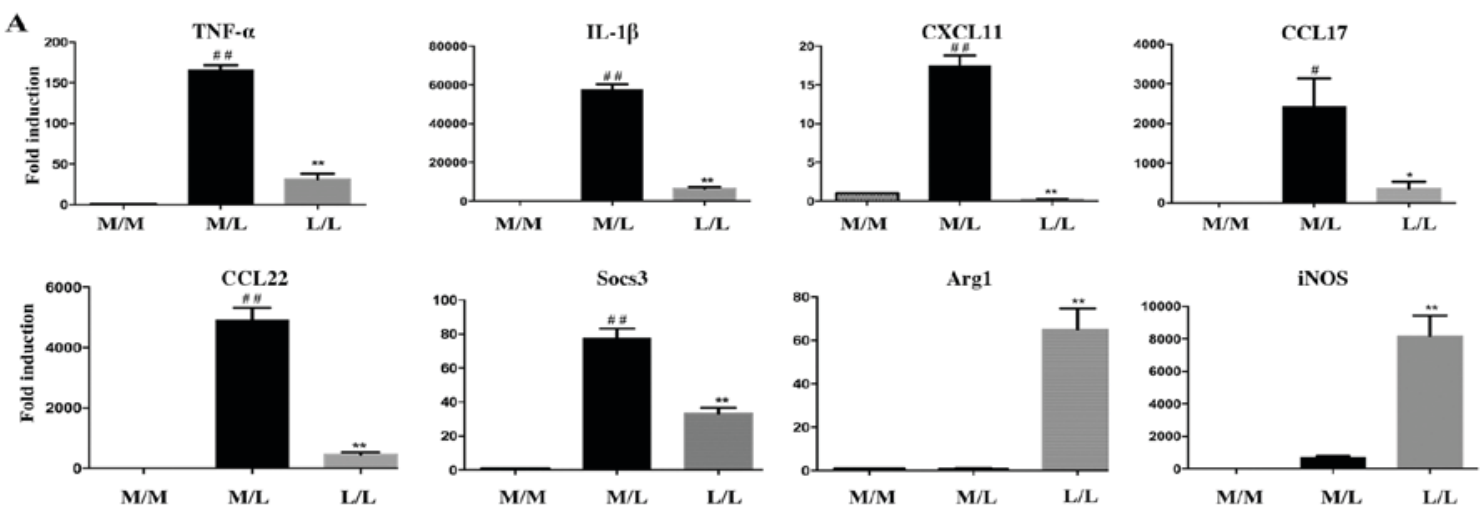

B

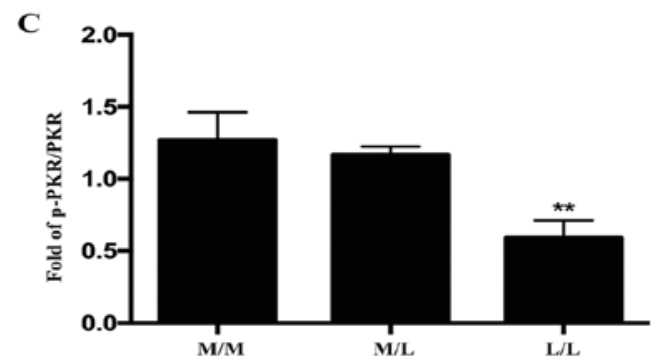

Figure 2. PKR inactivation is involved in the alterations in cytokine gene expression observed in LPS-tolerant macrophages. (A) Reverse transcription-quantitative polymerase chain reaction was performed to determine differences in the expression of inflammatory cytokine genes in LPS-tolerant L/L macrophages and LPS-activated M/L macrophages. The expression of the TNF- $\alpha$, IL-1 $\beta$, CXCL11, CCL17, CCL22 and Socs 3 mRNAs was markedly downregulated, while the expression of the Arg1 and iNOS mRNAs was upregulated, in LPS-tolerant L/L macrophages compared with LPS-activated M/L macrophages. (B) Representative western blot bands for the protein expression of p-PKR and PKR. $\beta$-actin was used as a loading control. (C) Quantification of the ratio of the intensities of the $\mathrm{p}-\mathrm{PKR} / \mathrm{PKR}$ bands by densitometry. ${ }^{* \mathrm{P}}<0.05$ and ${ }^{\# \#} \mathrm{P}<0.01$ vs. M/M cells; ${ }^{*} \mathrm{P}<0.05$ and ${ }^{* *} \mathrm{P}<0.01$ vs. $\mathrm{M} / \mathrm{L}$ cells. Data represent the results from three independent experiments. PKR, interferon-induced double-stranded RNA-dependent protein kinase; LPS, lipopolysaccharide; TNF- $\alpha$, tumor necrosis factor- $\alpha$; IL-1 $\beta$, interleukin-1 $\beta$; CXCL11, C-X-C motif chemokine ligand 11; CCL, C-C motif chemokine ligand; Socs3, suppressor of cytokine signaling 3; Arg1, arginase 1; iNOS, nitric oxide synthase 2; p-PKR, phosphorylated-PKR; M/M, initial incubation with medium followed by further incubation with medium; M/L, initial incubation with medium followed by LPS stimulation; L/L, initial incubation with LPS followed by restimulation with LPS.

Cytokine expression differs between L/L macrophages and $M / L$ macrophages. Cells were stimulated with or without LPS for $20 \mathrm{~h}$, washed twice with PBS and restimulated with LPS for $4 \mathrm{~h}$. Cells were subsequently lysed and RNA was isolated. The gene expression levels in RAW264.7 cells were detected by RT-qPCR. Levels of TNF- $\alpha$, IL-1 $\beta$, CXCL11, CCL17, CCL22 and Socs 3 mRNA were markedly decreased in LPS-tolerant $\mathrm{L} / \mathrm{L}$ macrophages compared with LPS-activated M/L macrophages (Fig. 2A). However, elevated levels of Arg1 and iNOS mRNA were detected in the LPS-tolerant L/L macrophages compared with LPS-activated $\mathrm{M} / \mathrm{L}$ macrophages.
PKR inactivation is involved in the altered cytokine gene expression observed in LPS-tolerant macrophages. Macrophages were cultured and stimulated with LPS as described above. Cells were lysed and protein levels were measured by western blotting at $2 \mathrm{~h}$ following the LPS rechallenge. RAW264.7 macrophages that were restimulated with LPS for $2 \mathrm{~h}$ after the initial $20 \mathrm{~h}$ challenge with LPS exhibited significant inactivation of PKR compared with cells challenged with LPS for only $2 \mathrm{~h}$ (Fig. 2B and C). However, the level of p-PKR was not statistically significantly different between $\mathrm{M} / \mathrm{M}$ and $\mathrm{M} / \mathrm{L}$ macrophages (Fig. $2 \mathrm{C}$ ). In addition, total PKR levels were not altered among the groups (Fig. 2B). 
A

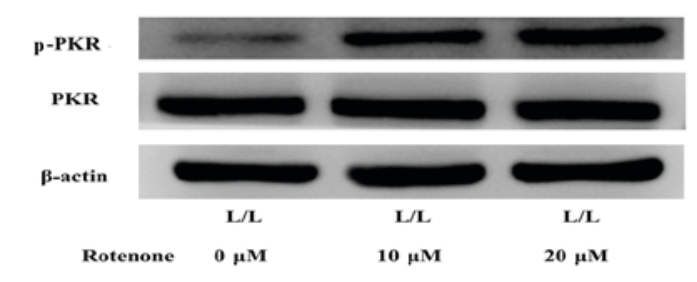

B

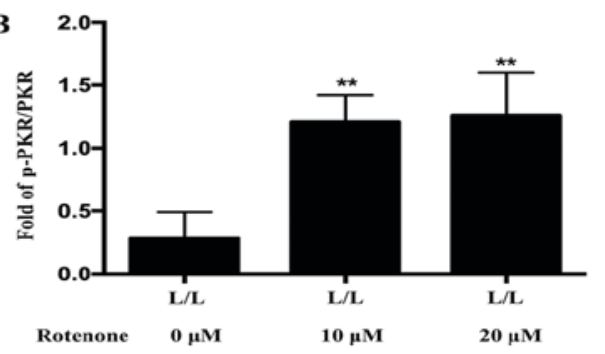

C
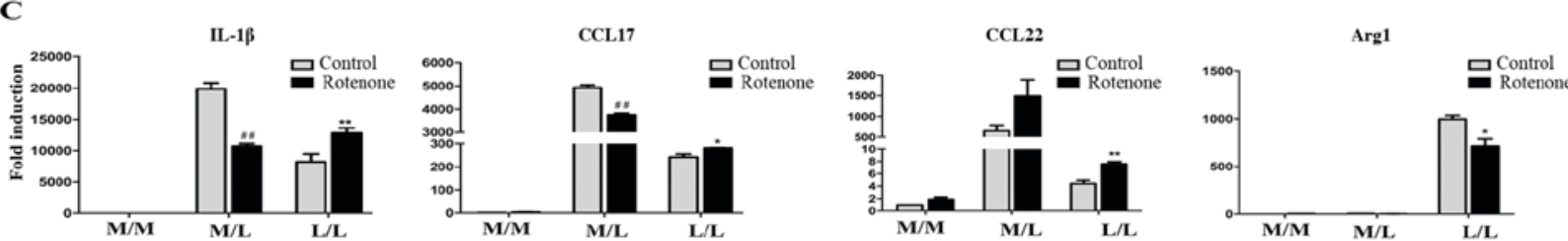

iNos

TNF- $\alpha$
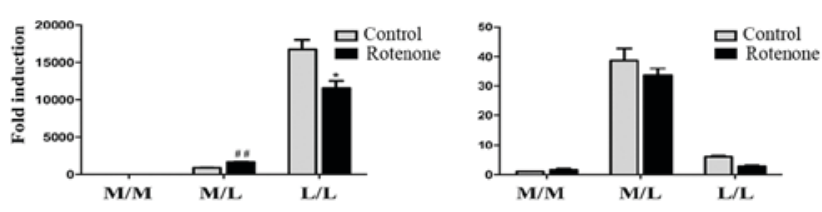

CXCL11
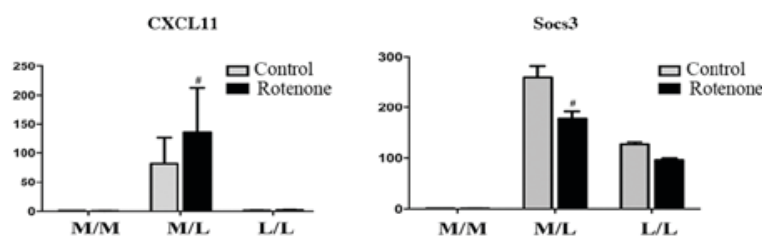

Figure 3. Rotenone ameliorates endotoxin tolerance by activating PKR. (A) Representative western blot bands for the protein expression of p-PKR and PKR. $\beta$-actin was used as the loading control. PKR activation was induced by 10 and $20 \mu \mathrm{M}$ rotenone in LPS-tolerant L/L RAW264.7 cells. (B) Quantification of the ratio of the intensities of the p-PKR/PKR bands by densitometry. The OD of the target protein is presented as a proportion of the $\beta$-actin OD. (C) Rotenone at a concentration of $10 \mu \mathrm{M}$ alleviated endotoxin tolerance by activating PKR. Reverse transcription-quantitative polymerase chain reaction results demonstrated increased levels of the IL-1 $\beta$, CCL17 and CCL22 mRNAs, and decreased levels of the Arg1 and iNOS mRNAs, in rotenone-treated LPS-tolerant L/L macrophages compared with untreated LPS-tolerant L/L macrophages. The expression of TNF- $\alpha$, CXCL11 and Socs3 mRNAs was not significantly different between the rotenone-treated and untreated LPS-tolerant $\mathrm{L} / \mathrm{L}$ macrophage groups. ${ }^{*} \mathrm{P}<0.05$ and ${ }^{\# \#} \mathrm{P}<0.01 \mathrm{vs}$. untreated/control M/L macrophages; ${ }^{*} \mathrm{P}<0.05$ and ${ }^{* *} \mathrm{P}<0.01$ vs. untreated/control L/L macrophages. Data represent the results from three independent experiments. PKR, interferon-induced double-stranded RNA-dependent protein kinase; p-PKR, phosphorylated-PKR; LPS, lipopolysaccharide; OD, optical density; IL-1 $\beta$, interleukin-1 $\beta$; CCL, C-C motif chemokine ligand; Arg1, arginase 1; iNOS, nitric oxide synthase 2; TNF- $\alpha$, tumor necrosis factor- $\alpha$; CXCL11, C-X-C motif chemokine ligand 11; Socs3, suppressor of cytokine signaling 3; M/M, initial incubation with medium followed by further incubation with medium; M/L, initial incubation with medium followed by LPS stimulation; L/L, initial incubation with LPS followed by restimulation with LPS.

Rotenone alleviates endotoxin tolerance by activating PKR in RAW264.7 cells. It has been previously demonstrated that rotenone activates PKR (33). The level of p-PKR was markedly increased following treatment with rotenone $(10$ or $20 \mu \mathrm{M})$ in LPS-tolerant L/L macrophages compared with untreated LPS-tolerant L/L macrophages (Fig. 3A and B). In addition, the level of p-PKR was not statistically significantly different between LPS-tolerant L/L macrophages treated with 10 and $20 \mu \mathrm{M}$ rotenone. Furthermore, the mRNA levels of IL-1 $\beta$, CCL17 and CCL22 were increased, while the mRNA levels of the Arg1 and iNOS were decreased, in rotenone-treated LPS-tolerant L/L macrophages compared with untreated LPS-tolerant L/L macrophages (Fig. 3C). The levels of TNF- $\alpha$, CXCL11 and Socs3 mRNA were not statistically significantly different between rotenone-treated and untreated LPS-tolerant L/L macrophage groups.

PKR mediates macrophage reprogramming in LPS-tolerant RAW264.7 cells by inactivating AKT. RAW264.7 cells were cultured in DMEM and stimulated with LPS as described above. Following a 2-h restimulation with LPS, macrophages were lysed and levels of proteins were measured by western blotting. AKT was activated in LPS-activated M/L macrophages compared with $\mathrm{M} / \mathrm{M}$ macrophages that received no stimulation with LPS (Fig. 4A and B). However, the levels of p-AKT were markedly decreased in LPS-tolerant L/L macrophages compared with LPS-activated M/L macrophages (Fig. 4A and B). The total AKT levels were not altered among the groups (Fig. 4A). Rotenone induces PKR phosphorylation. In the present study, AKT was activated in rotenone-treated LPS-tolerant L/L macrophages compared with the untreated L/L macrophages (Fig. 4C and D). Ly294002, a phosphatidylinositol-4,5-bisphosphate 3-kinase (PI3K)-AKT inhibitor, was added to LPS-tolerant L/L cells prior to the $1 \mathrm{~h}$ rotenone treatment. Ly294002 $(10 \mu \mathrm{M})$ did not affect the activation of PKR in rotenone-treated LPS-tolerant L/L macrophages (Fig. 5). However, AKT activation in rotenone-treated LPS-tolerant L/L macrophages was inhibited by Ly 294002 (Fig. 6A and B). Furthermore, Ly294002 partially reversed the rotenone-induced variations in gene expression in LPS-tolerant L/L macrophages (Fig. 6C). Specifically, Ly294002 downregulated IL-1 $\beta$ and CCL22 expression and upregulated Arg1 and iNOS expression in the rotenone-treated LPS-tolerant L/L macrophages (Fig. 6C).

\section{Discussion}

Following long-term exposure to LPS, macrophages enter an immunosuppressive state and are unable to respond to further LPS challenges. The immunosuppressive or 
A

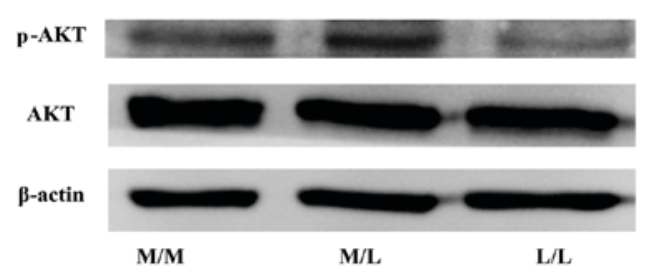

C

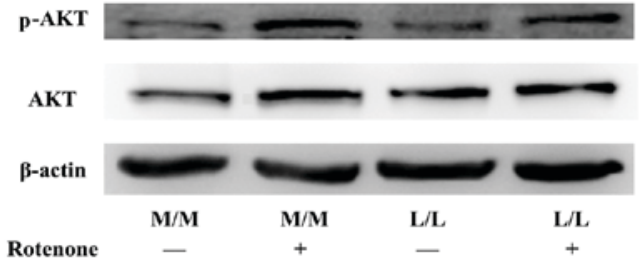

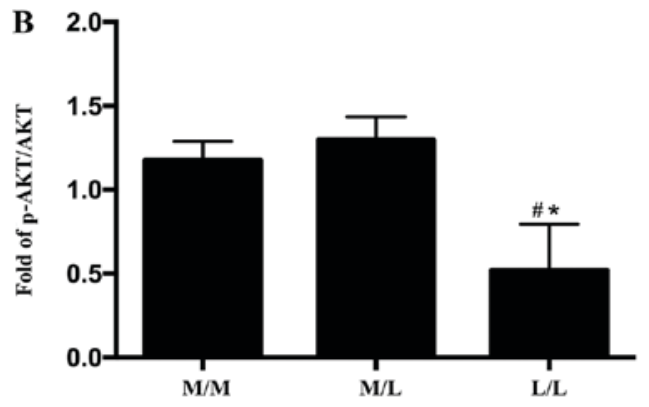

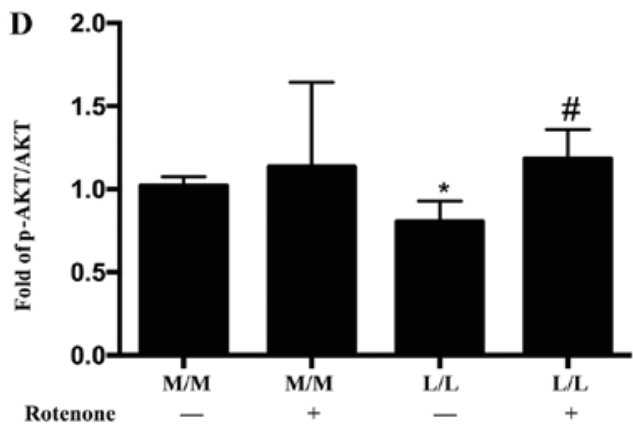

Figure 4. PKR mediates macrophage reprogramming in LPS-tolerant RAW264.7 macrophages by inactivating AKT. (A) Representative western blot bands for the protein expression of $\mathrm{p}-\mathrm{AKT}$ and AKT in LPS-activated M/L and LPS-tolerant L/L macrophages. $\beta$-actin was used as the loading control. (B) Quantification of the ratio of the intensities of the p-AKT/AKT bands by densitometry. ${ }^{*} \mathrm{P}<0.05$ vs. M/M group; $\mathrm{P}<0.05$ vs. M/L group. (C) Representative western blot bands for $\mathrm{p}$-AKT and AKT protein expression in rotenone-treated and untreated LPS-tolerant L/L macrophages. $\beta$-actin was used as the loading control. (D) Quantification of the ratio of the intensities of the p-AKT/AKT bands by densitometry. ${ }^{*} \mathrm{P}<0.05$ vs. untreated $\mathrm{M} / \mathrm{M}$ group; ${ }^{\#} \mathrm{P}<0.05$ vs. untreated L/L group. Data represent the results from three independent experiments. PKR, interferon-induced double-stranded RNA-dependent protein kinase; LPS, lipopolysaccharide; p-AKT, phosphorylated-AKT; M/M, initial incubation with medium followed by further incubation with medium; M/L, initial incubation with medium followed by LPS stimulation; L/L, initial incubation with LPS followed by restimulation with LPS.

hyporesponsive state that develops is termed endotoxin tolerance (5). Endotoxin tolerance has been associated with various diseases, including sepsis, trauma, pancreatitis and acute coronary syndrome $(15,34,35)$. The current hypothesis regarding the host immune response in patients with sepsis indicates that it is characterized by an initial hyperinflammatory phase that is sustained over several days and progresses into a protracted immunosuppressive phase, indicating that macrophages enter a tolerant state $(18,36)$. In patients with sepsis, mortality occurs primarily due to the development of uncontrolled secondary infections as a result of immunosuppression (37-39). Therefore, strategies that prevent endotoxin tolerance have become a topic of interest in therapies for sepsis (39).

In the present study, RAW264.7 macrophage cells were stimulated with $100 \mathrm{ng} / \mathrm{ml}$ LPS for $20 \mathrm{~h}$, washed twice with PBS and restimulated with $100 \mathrm{ng} / \mathrm{ml} \mathrm{LPS}$ for 2 or $4 \mathrm{~h}$ to establish an LPS-tolerant model, as described previously $(9,11)$. TNF- $\alpha$ levels have been reported to be significantly decreased in tolerant macrophages and are considered a reliable marker of endotoxin tolerance $(6,40,41)$. In the present study, TNF- $\alpha$ secretion from LPS restimulated tolerant macrophages was markedly decreased compared with LPS-activated macrophages, indicating that the endotoxin tolerance model was successfully established.

In LPS tolerant macrophages, the expression of cytokine genes is reprogrammed rather than inhibited $(5,12,13)$. During macrophage reprogramming, the expression of certain genes is downregulated, while other genes are upregulated (42). This phenomenon is similar to macrophage polarization, in which macrophages undergo polarized differentiation into classically activated macrophages (M1) or alternatively activated macrophages (M2) in response to different stimuli (43). M1 macrophages are characterized by increased production of proinflammatory cytokines, nitric oxide and reactive oxygen species that mediate antimicrobial activities and induce cellular immunity $(44,45)$. M2 macrophages are characterized by intracellular expression of Arg1 and secretion of chitinases, including Chil3, and anti-inflammatory cytokines, including interleukin-10 (46). Therefore, M2 macrophages have been associated with helminthic infection and tissue repair (47). Macrophage tolerance and M2 polarization are associated processes. It was previously reported that the expression of M2-associated cytokines (CCL17, CCL22 and Arg1) was upregulated, while the expression of M1-associated cytokines (TNF- $\alpha$, IL-1 $\beta$, CXCL-11, Socs3 and iNOS) was downregulated, in LPS-tolerant macrophages (31). In the present study, the mRNA levels of the M1-associated cytokines TNF- $\alpha$, IL-1 $\beta$, CXCL-11 and Socs 3 were decreased and the levels of the M2-associated mediator Arg1 was increased, similar to M2 polarization. However, the levels of the M2-associated mediators CCL-17 and CCL-22 were decreased and the level of the M1-associated mediator iNOS was increased in 
A

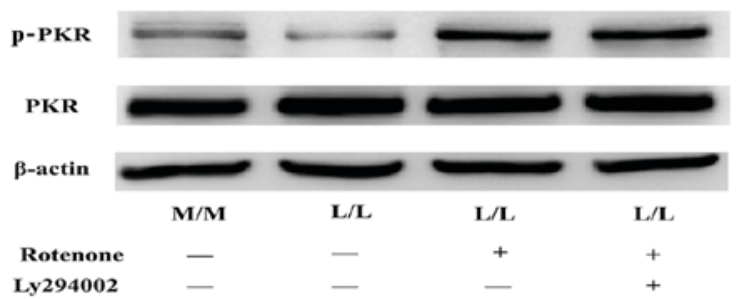

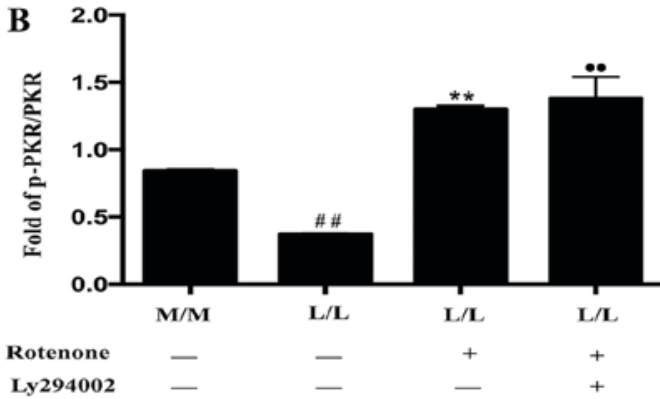

Figure 5. Ly294002 does not affect the activation of PKR in LPS-tolerant L/L macrophages. (A) Representative western blot bands for p-PKR and PKR protein expression following rotenone treatment with or without Ly294002 in LPS-tolerant L/L macrophages. $\beta$-actin was used as the loading control. (B) Quantification of the ratio of the intensities of the p-PKR/PKR bands by densitometry. ${ }^{\# \#} \mathrm{P}<0.01 \mathrm{vs}$. M/M (-/) macrophages, ${ }^{* * *} \mathrm{P}<0.01 \mathrm{vs}$. L/L (-/-) macrophages and ${ }^{~ " P}<0.01$ vs. L/L (-/-) macrophages. Data represent the results from three independent experiments. PKR, interferon-induced double-stranded RNA-dependent protein kinase; LPS, lipopolysaccharide; p-PKR, phosphorylated-PKR; M/M, initial incubation with medium followed by further incubation with medium; L/L, initial incubation with LPS followed by restimulation with LPS.

A

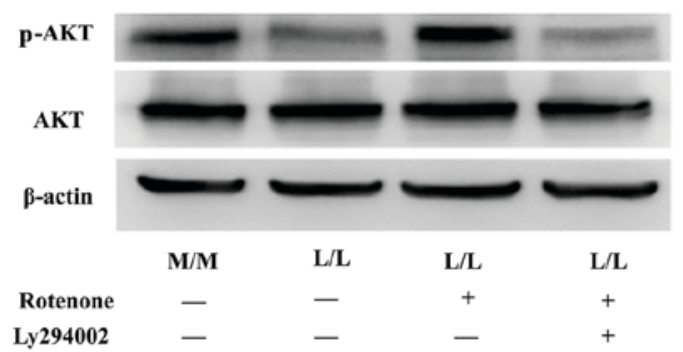

C
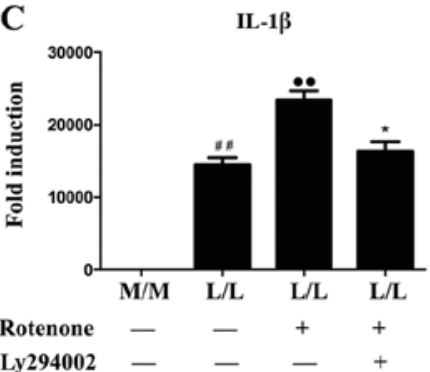

CCL22
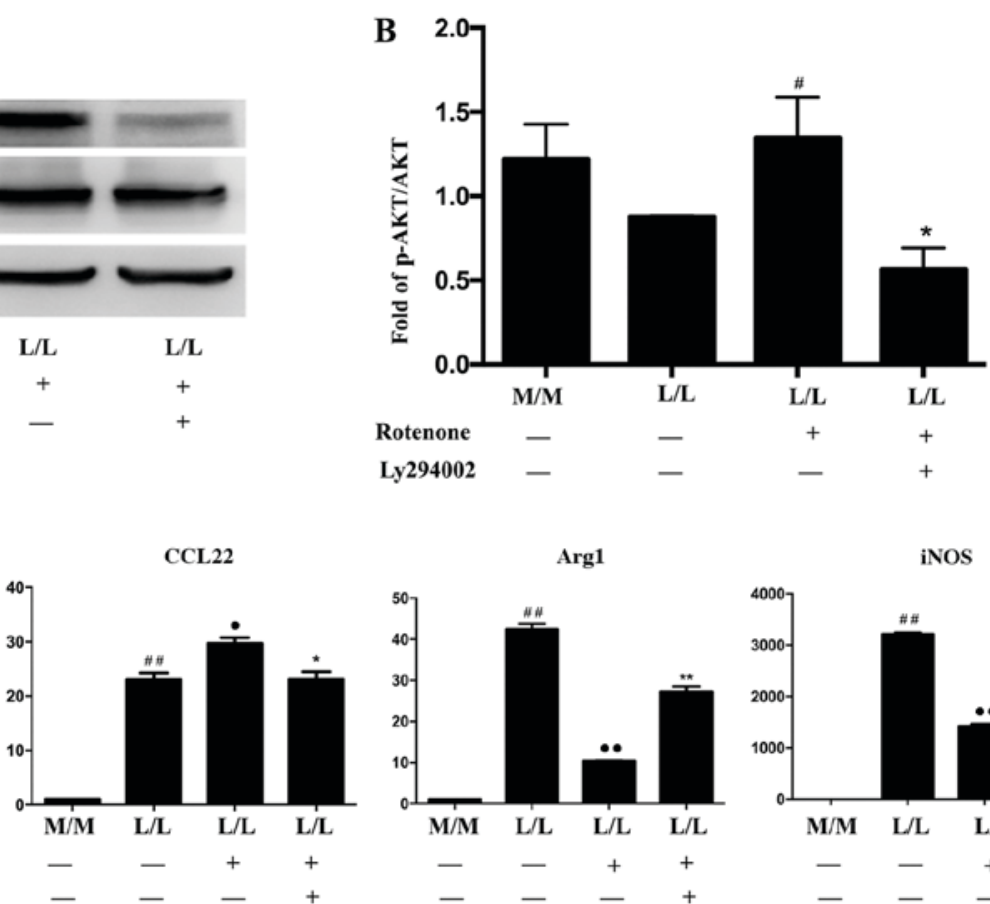

Arg1

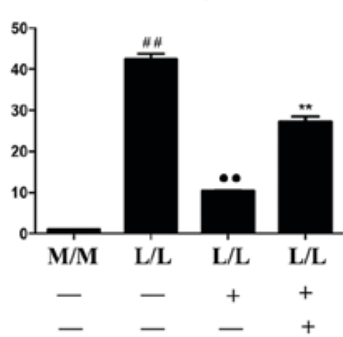

iNOS

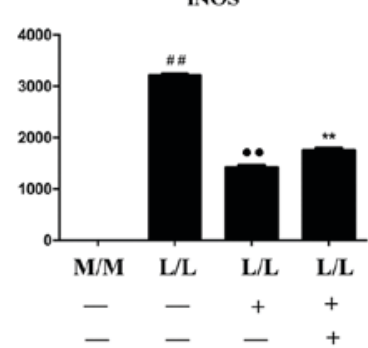

Figure 6. Ly294002 partially prevents the alterations in gene expression induced by rotenone in LPS-tolerant L/L macrophages. (A) Representative western blot bands for p-AKT and AKT protein expression following rotenone treatment with or without Ly294002 in LPS-tolerant L/L macrophages. $\beta$-actin was used as the loading control. (B) Quantification of the ratio of the intensities of the p-AKT/AKT bands by densitometry. ${ }^{*} \mathrm{P}<0.05$ vs. $\mathrm{L} / \mathrm{L}(-/-)$ macrophages, ${ }^{*} \mathrm{P}<0.05$ vs. L/L (+/-) macrophages. (C) Reverse transcription-quantitative polymerase chain reaction results demonstrated that Ly294002 induced downregulation of IL-1 $\beta$ and CCL22 expression, and upregulation of Arg1 and iNOS expression, in rotenone-treated LPS-tolerant L/L macrophages. ${ }^{\# \#} \mathrm{P}<0.01 \mathrm{vs}$. M/M (-/-) macrophages. ${ }^{\circ} \mathrm{P}<0.05$ and ${ }^{~} \mathrm{P}<0.01$ vs. $\mathrm{L} / \mathrm{L}(-/-)$ macrophages; ${ }^{*} \mathrm{P}<0.05$ and ${ }^{* *} \mathrm{P}<0.01 \mathrm{vs}$. $\mathrm{L} / \mathrm{L}(+/-)$ macrophages. Data represent the results from three independent experiments. LPS, lipopolysaccharide; p-AKT, phosphorylated-AKT; IL-1 $\beta$, interleukin-1 $\beta$; CCL22, C-C motif chemokine ligand 22; Arg1, arginase 1; iNOS, nitric oxide synthase $2 ; \mathrm{M} / \mathrm{M}$, initial incubation with medium followed by further incubation with medium; L/L, initial incubation with LPS followed by restimulation with LPS.

LPS-tolerant macrophages, which differed from M2 polarization. Variation in the expression of iNOS has been reported in LPS-tolerant cells as certain studies have demonstrated that it was elevated $(48,49)$, while others detected decreased iNOS levels, in LPS-tolerant cells $(10,13)$. These variations may depend on the cell type, duration of LPS stimulation and the concentration of the LPS used in the different studies.

In addition to its antiviral properties, $\mathrm{PKR}$ also participates in the regulation of inflammatory cytokine and chemokine expression, including IL-1 $\beta$, IL-18 and high-mobility group box 1 , by affecting transcription factors (25-27,33). Total PKR levels in tolerant macrophages were reported to be decreased through differential K63/K48 ubiquitination (50). However, the role of PKR in macrophage reprogramming remains to be elucidated. In the present study, p-PKR levels were markedly decreased in LPS-tolerant macrophages, whereas total PKR levels remained unaltered. Rotenone is a plant extract that activates PKR (33). Administration of rotenone in the present 
study regulated the mRNA expression of IL-1 $\beta$, CCL17, CCL22, Arg1 and iNOS in LPS-tolerant macrophages. Based the above data, it may be hypothesized that PKR activation partially reverses macrophage reprogramming in endotoxin tolerance. However, the expression of the TNF- $\alpha$, CXCL11 and Socs 3 mRNAs was not significantly different between rotenone-treated and untreated LPS-tolerant cells. The expression of these cytokines may not be regulated by PKR. However, the expression of these cytokines has been previously demonstrated to be regulated by other proteins, including p 21 and p50 (11).

It has been demonstrated that several signaling pathways, including NF-kb $(51,52)$ and mitogen-activated protein kinase $(29,53)$ pathways, are regulated by PKR to promote cytokine and chemokine production. PKR has also been reported to participate in physiological activities, including coordinating skeletal muscle differentiation and choroidal neovascularization, via the PI3K/AKT signaling pathway $(54,55)$. However, to the best of our knowledge, it has not been previously determined whether PKR mediates macrophage reprogramming via the PI3K/AKT signaling pathway. In the present study, AKT was inactivated in LPS-tolerant macrophages. Rotenone-induced PKR activation was demonstrated to increase the level of p-AKT in LPS-tolerant cells, reversing endotoxin tolerance-induced inactivation of AKT. Furthermore, inhibition of PI3K-AKT signaling with Ly294002, a PI3K/AKT inhibitor, partially reversed the rotenone-induced alleviation of endotoxin tolerance, which was supported by the alterations in the expression of several endotoxin tolerance-associated genes, including IL-1 $\beta$, CCL22, Arg1 and iNOS.

In conclusion, the results of the current study demonstrated that PKR inhibition induced endotoxin tolerance in macrophages and these effects were partially mediated by the PI3K/AKT signaling pathway. Therefore, PKR may be a potential target for the treatment of endotoxin tolerance.

\section{Acknowledgements}

Not applicable.

\section{Funding}

The present study was funded by grants from the Natural Science Foundation of Guangdong Province (grant no. 2016A030313269) and Fundamental Research Funds for the Central Universities (grant no. 15ykpy14).

\section{Availability of data and materials}

The datasets used and/or analyzed during the current study are available from the corresponding author on reasonable request.

\section{Authors' contributions}

JW and XG conceived and designed the experiments. HX, JC, MC, FP and CQ performed the experiments. HX and XS analyzed the data and produced the pictures. HX and CQ produced the manuscript. HX submitted the manuscript and revised it. All authors read and approved the final manuscript.

\section{Ethics approval and consent to participate}

Not applicable.

\section{Consent for publication}

Not applicable.

\section{Competing interests}

The authors declare that they have no competing interests.

\section{References}

1. Gordon S and Taylor PR: Monocyte and macrophage heterogeneity. Nat Rev Immunol 5: 953-964, 2005.

2. Lawrence T and Natoli G: Transcriptional regulation of macrophage polarization: Enabling diversity with identity. Nat Rev Immunol 11: 750-761, 2011.

3. Beutler B: SHIP, TGF-beta, and endotoxin tolerance. Immunity 21: 134-135, 2004.

4. O'Neill LA and Bowie AG: The family of five: TIR-domain-containing adaptors in Toll-like receptor signalling. Nat Rev Immunol 7: 353-364, 2007.

5. Cavaillon JM and Adib-Conquy M: Bench-to-bedside review: Endotoxin tolerance as a model of leukocyte reprogramming in sepsis. Crit Care 10: 233, 2006.

6. Fan H and Cook JA: Molecular mechanisms of endotoxin tolerance. J Endotoxin Res 10: 71-84, 2004.

7. del Fresno C, García-Rio F, Gómez-Piña V, Soares-Schanoski A, Fernández-Ruíz I, Jurado T, Kajiji T, Shu C, Marín E, Gutierrez del Arroyo A, et al: Potent phagocytic activity with impaired antigen presentation identifying lipopolysaccharide-tolerant human monocytes: Demonstration in isolated monocytes from cystic fibrosis patients. J Immunol 182: 6494-6507, 2009.

8. Zuckerman SH and Evans GF: Endotoxin tolerance: In vivo regulation of tumor necrosis factor and interleukin-1 synthesis is at the transcriptional level. Cell Immunol 140: 513-519, 1992.

9. Rajaiah R, Perkins DJ, Polumuri SK, Zhao A, Keegan AD and Vogel SN: Dissociation of endotoxin tolerance and differentiation of alternatively activated macrophages. J Immunol 190: 4763-4772, 2013

10. Piao W, Song C, Chen H, Diaz MA, Wahl LM, Fitzgerald KA, Li L and Medvedev AE: Endotoxin tolerance dysregulates MyD88- and Toll/IL-1R domain-containing adapter inducing IFN-beta-dependent pathways and increases expression of negative regulators of TLR signaling. J Leukoc Biol 86: 863-875, 2009.

11. RackovG,Hernández-JiménezE,ShokriR,Carmona-RodríguezL, Mañes S, Álvarez-Mon M, López-Collazo E, Martínez-A C and Balomenos D: p21 mediates macrophage reprogramming through regulation of $\mathrm{p} 50-\mathrm{p} 50 \mathrm{NF}-\kappa \mathrm{B}$ and IFN- $\beta$. J Clin Invest 126: 3089-3103, 2016.

12. Foster SL and Medzhitov R: Gene-specific control of the TLR-induced inflammatory response. Clin Immunol 130: 7-15, 2009.

13. Foster SL, Hargreaves DC and Medzhitov R: Gene-specific control of inflammation by TLR-induced chromatin modifications. Nature 447: 972-978, 2007.

14. Shalova IN, Lim JY, Chittezhath M, Zinkernagel AS, Beasley F, Hernández-Jiménez E, Toledano V, Cubillos-Zapata C, Rapisarda A Chen J, et al: Human monocytes undergo functional re-programming during sepsis mediated by hypoxia-inducible factor-1 $\alpha$. Immunity 42: 484-498, 2015.

15. Pena OM, Hancock DG, Lyle NH, Linder A, Russell JA, Xia J, Fjell CD, Boyd JH and Hancock RE: An endotoxin tolerance signature predicts sepsis and organ dysfunction at initial clinical presentation. EBioMedicine 1: 64-71, 2014.

16. Hotchkiss RS, Levy JH and Levi M: Sepsis-induced disseminated intravascular coagulation, symmetrical peripheral gangrene, and amputations. Crit Care Med 41: e290-e291, 2013.

17. Moller K: Of cells and men: Ex vivo and in vivo tolerance to lipopolysaccharide. Crit Care Med 39: 1997-1998, 2011.

18. Angus DC and van der Poll T: Severe sepsis and septic shock. N Engl J Med 369: 2063, 2013. 
19. Delano MJ and Ward PA: Sepsis-induced immune dysfunction: Can immune therapies reduce mortality? J Clin Invest 126: 23-31, 2016.

20. Lopez-Collazo E and del Fresno C: Pathophysiology of endotoxin tolerance: Mechanisms and clinical consequences. Crit Care 17: 242, 2013.

21. Sly LM, Rauh MJ, Kalesnikoff J, Song CH and Krystal G: LPS-induced upregulation of SHIP is essential for endotoxin tolerance. Immunity 21: 227-239, 2004.

22. Xiong Y and Medvedev AE: Induction of endotoxin tolerance in vivo inhibits activation of IRAK4 and increases negative regulators IRAK-M, SHIP-1, and A20. J Leukoc Biol 90: 1141-1148, 2011.

23. Meurs E, Chong K, Galabru J, Thomas NS, Kerr IM, Williams BR and Hovanessian AG: Molecular cloning and characterization of the human double-stranded RNA-activated protein kinase induced by interferon. Cell 62: 379-390, 1990.

24. Shen SJ, Zhang YH, Gu XX, Jiang SJ and Xu LJ: Yangfei Kongliu Formula, a compound Chinese herbal medicine, combined with cisplatin, inhibits growth of lung cancer cells through transforming growth factor- $\beta 1$ signaling pathway. J Integr Med 15: 242-251, 2017.

25. Balachandran $\mathrm{S}$ and Barber GN: PKR in innate immunity, cancer, and viral oncolysis. Methods Mol Biol 383: 277-301, 2007.

26. Williams BR: Signal integration via PKR. Sci STKE 2001: re2, 2001.

27. García MA, Gil J, Ventoso I, Guerra S, Domingo E, Rivas C and Esteban M: Impact of protein kinase PKR in cell biology: From antiviral to antiproliferative action. Microbiol Mol Biol Rev 70 1032-1060, 2006.

28. Zhang $\mathrm{P}$ and Samuel CE: Induction of protein kinase PKR-dependent activation of interferon regulatory factor 3 by vaccinia virus occurs through adapter IPS-1 signaling. J Biol Chem 283: 34580-34587, 2008

29. Zamanian-Daryoush M, Mogensen TH, DiDonato JA and Williams BR: NF-kappaB activation by double-stranded-RNA-activated protein kinase (PKR) is mediated through NF-kappaB-inducing kinase and IkappaB kinase. Mol Cell Biol 20: 1278-1290, 2000.

30. Han BH, Lee YJ, Yoon JJ, Choi ES, Namgung S, Jin XJ, Jeong DH, Kang DG and Lee HS: Hwangryunhaedoktang exerts anti-inflammation on LPS-induced NO production by suppressing MAPK and NF- $\kappa \mathrm{B}$ activation in RAW264.7 macrophages. J Integr Med 15: 326-336, 2017.

31. Porta C, Rimoldi M, Raes G, Brys L, Ghezzi P, Di Liberto D, Dieli F, Ghisletti S, Natoli G, De Baetselier P, et al: Tolerance and M2 (alternative) macrophage polarization are related processes orchestrated by p50 nuclear factor kappaB. Proc Natl Acad Sci USA 106: 14978-14983, 2009.

32. Livak KJ and Schmittgen TD: Analysis of relative gene expression data using real-time quantitative PCR and the 2(-Delta Delta C(T)) method. Methods 25: 402-408, 2001.

33. Lu B, Nakamura T, Inouye K, Li J, Tang Y, Lundbäck P, Valdes-Ferrer SI, Olofsson PS, Kalb T, Roth J, et al: Novel role of PKR in inflammasome activation and HMGB1 release. Nature 488: 670-674, 2012

34. Cavaillon JM, Adrie C, Fitting C and Adib-Conquy M: Endotoxin tolerance: Is there a clinical relevance? J Endotoxin Res 9: 101-107, 2003.

35. del Fresno C, Gómez-Piña V, Lores V, Soares-Schanoski A, Fernández-Ruiz I, Rojo B, Alvarez-Sala R, Caballero-Garrido E, García F, Veliz T, et al: Monocytes from cystic fibrosis patients are locked in an LPS tolerance state: Down-regulation of TREM-1 as putative underlying mechanism. PLoS One 3: e2667, 2008 .
36. Escoll P, del Fresno C, García L, Vallés G, Lendínez MJ, Arnalich F and López-Collazo E: Rapid up-regulation of IRAK-M expression following a second endotoxin challenge in human monocytes and in monocytes isolated from septic patients. Biochem Biophys Res Commun 311: 465-472, 2003

37. Hotchkiss RS, Monneret G and Payen D: Immunosuppression in sepsis: A novel understanding of the disorder and a new therapeutic approach. Lancet Infect Dis 13: 260-268, 2013.

38. Hotchkiss RS and Karl IE: The pathophysiology and treatment of sepsis. N Engl J Med 348: 138-150, 2003.

39. Hotchkiss RS and Opal S: Immunotherapy for sepsis-a new approach against an ancient foe. N Engl J Med 363: 87-89, 2010.

40. Nahid MA, Satoh M and Chan EK: MicroRNA in TLR signaling and endotoxin tolerance. Cell Mol Immunol 8: 388-403, 2011.

41. West MA and Koons A: Endotoxin tolerance in sepsis: Concentration-dependent augmentation or inhibition of LPS-stimulated macrophage TNF secretion by LPS pretreatment. J Trauma 65: 893-898, 2008

42. Biswas SK and Lopez-Collazo E: Endotoxin tolerance: New mechanisms, molecules and clinical significance. Trends Immunol 30: 475-487, 2009.

43. Mosser DM and Edwards JP: Exploring the full spectrum of macrophage activation. Nat Rev Immunol 8: 958-969, 2008.

44. Mantovani A, Sozzani S, Locati M, Allavena P and Sica A: Macrophage polarization: Tumor-associated macrophages as a paradigm for polarized M2 mononuclear phagocytes. Trends Immunol 23: 549-555, 2002.

45. Mosser DM: The many faces of macrophage activation. J Leukoc Biol 73: 209-212, 2003

46. Shirey KA, Pletneva LM, Puche AC, Keegan AD, Prince GA, Blanco JC and Vogel SN: Control of RSV-induced lung injury by alternatively activated macrophages is IL-4R alpha-, TLR4-, and IFN-beta-dependent. Mucosal Immunol 3: 291-300, 2010.

47. Gordon S: Alternative activation of macrophages. Nat Rev Immunol 3: 23-35, 2003.

48. Castegren M, Skorup P, Lipcsey M, Larsson A and Sjolin J: Endotoxin tolerance variation over $24 \mathrm{~h}$ during porcine endotoxemia: Association with changes in circulation and organ dysfunction. PLoS One 8: e53221, 2013.

49. Dias MB, Almeida MC, Carnio EC and Branco LG: Role of nitric oxide in tolerance to lipopolysaccharide in mice. J Appl Physiol (1985) 98: 1322-1327, 2005.

50. Perkins DJ, Qureshi N and Vogel SN: A Toll-like receptor-responsive kinase, protein kinase $\mathrm{R}$, is inactivated in endotoxin tolerance through differential K63/K48 ubiquitination. MBio 1: e00239-e002310, 2010.

51. Goh KC, deVeer MJ and Williams BR: The protein kinase PKR is required for p38 MAPK activation and the innate immune response to bacterial endotoxin. EMBO J 19: 4292-4297, 2000.

52. Nakamura T, Furuhashi M, Li P, Cao H, Tuncman G, Sonenberg N, Gorgun CZ and Hotamisligil GS: Double-stranded RNA-dependent protein kinase links pathogen sensing with stress and metabolic homeostasis. Cell 140: 338-348, 2010.

53. Bonnet MC, Weil R, Dam E, Hovanessian AG and Meurs EF: PKR stimulates NF-kappaB irrespective of its kinase function by interacting with the IkappaB kinase complex. Mol Cell Biol 20: 4532-4542, 2000.

54. Alisi A, Spaziani A, Anticoli S, Ghidinelli M and Balsano C: PKR is a novel functional direct player that coordinates skeletal muscle differentiation via p38MAPK/AKT pathways. Cell Signal 20: 534-542, 2008.

55. Zhu M, Liu X, Wang S, Miao J, Wu L, Yang X, Wang Y, Kang L, $\mathrm{Li} \mathrm{W}$, Cui C, et al: PKR promotes choroidal neovascularization via upregulating the PI3K/Akt signaling pathway in VEGF expression. Mol Vis 22: 1361-1374, 2016. 